7th International Symposium on Superalloy 718 and Derivatives Edited by: E.A. Ott, J.R. Groh, A. Banik, I. Dempster, T.P. Gabb, R. Helmink, X. Liu, A. Mitchell, G.P. Sjöberg, and A. Wusatowska-Sarnek TMS (The Minerals, Metals \& Materials Society), 2010

\title{
MODELING THE HOT FORGING OF NICKEL-BASED SUPERALLOYS: IN718 and ALLOY 718PLUS
}

\author{
Esteban B. Marin ${ }^{1}$, Douglas J. Bammann ${ }^{1}$, Arthur Brown ${ }^{2}$, Haitham El Kadiri ${ }^{1}$, R. Daniel \\ Costley $^{3}$, Paul T. Wang ${ }^{1}$, Mark F. Horstemeyer ${ }^{1}$ \\ ${ }^{1}$ Center for Advanced Vehicular Systems, Mississippi State University, MS, 39759 \\ ${ }^{2}$ Sandia National Laboratories, Livermore, CA, 94550 \\ ${ }^{3}$ Miltec Research \& Technology, Oxford, MS, 38655
}

Keywords: Material Model, Internal State Variable, Forging, Inconel Superalloys

\begin{abstract}
An internal state variable material model is used to describe the rate- and temperaturedependent large deformation response of the nickel-based superalloys Inconel 718 and Inconel 718 Plus. The current version of the material model describes the elastic-plastic and thermal deformation of metals, having two internal state variables whose evolution equations account for dislocation hardening and static/dynamic recovery processes. Other microstructural features such as recrystallization and grain growth are currenlty being added to the model. Experimental data from mechanical characterization tests of cylindrical and double cone compression specimens are used, respectively, to calibrate the material model and to validate its predictive capability. In general, the calibrated model predicts well the experimental stress/load levels as well as the rate and temperature dependence of the mechanical response of these superalloys.
\end{abstract}

\section{Introduction}

The superalloys IN718 and IN718Plus have been very well characterized in terms of their chemistry, microstructure (precipitation phases), manufacturing processing and mechanical properties $[1,2,3,4]$ It is well known that the use of the correct chemical components together with an adequate thermo-mechanical processing (deformation processing, heat treatment, aging) develop in these materials the desirable microstructure (precipitate phases) that give these alloys their good elevated temperature strentgh, thermal stability, and hot workability, characteristics needed for the long-term high-temperature environments typical of aircraft engine turbine parts [5]. However, there is still a demand to understand the mechanical behavior at the final stages of the manufacturing process, which is typically a multi-step hot forging process [6].

During hot forging processes, the microstructure and mechanical behavior of these superalloys tpically change by metallurgical transformations. Microstructural features such as dislocation structures, annealing phenomena (recovery, recrystallization and grain growth), and precipitate phases are mainly responsible for the final mechanical properties of the material [7], and hence for the performance of the manufactured part during service. In this context, much research has been directed at understanding the mechanisms and phenomenology of microstructure evolution during hot deformation. Microstructural processes such as dynamic, metadynamic and static recrystallization as well as grain growth in metals [8], and their relation to the hot processing parameters has been studied and modeled in these superalloys, with the bulk of the study mainly concentrated on IN718, an alloy invented almost a half-century ago $[9,10,11,12,13,14,15]$. Modeling the hot deformation of superalloys during 
thermo-mechanical processing implies the implementation of microstructural models in numerical codes and its application to solve deformation processing problems. In this regard, many studies has been published in the literature, where the connection between processing parameters and microstructure evolution has been made [16,17]. In a more consistent approach, from a constitutive modeling point of view, the above microstructural models should be included in a general constitutive framework that describes the high-temperature large-deformation behavior of these superalloys. Such formulations typically rely on the use of internal state variable (ISV) models consistently formulated using kinematics and thermodynamics formalisms. In this case, specific microstructural variables, such as dislocation density, fraction of recrystallized materials and grain size, can be identified as part of the state variables of the model and whose evolution equations will capture the physics of deformation. In this context, many ISV models for metals exist [18] and a number of them have been used to predict some features of the microstructure evolution (dislocation hardening/recovery, recrystallization, grain growth) and mechanical properties during hot deformation $[19,20,21]$, with some of these models having been applied to model the hot forging response of IN718 [22]. Particularly, a modification of the BCJ model [18] is used in this work to represent the rate- and temperature-dependent response of the Inconel superalloys.

\section{Experimental Results}

An experimental program carried out at the Air Force Research Lab [23] scheduled a number of isothermal compression (upsetting) tests on cylindrical and double truncated cone specimens under various strain rates and temperatures to generate the needed experimental data for development of constitutive models for IN718 and IN718Plus. The test matrices used for these mechanical characterization studies are presented in Tables 1 and 2. The cylindrical specimens had a diameter and heigth of approx. $8.4 \mathrm{~mm}$ and $12.7 \mathrm{~mm}$, respectively, while the geometry of the double truncated cone specimes is presented in Fig.1A

The compression tests of the cylindrical specimens generated true strain-true stress curves that can be used to fit the predicted response of a rate- and temperature-dependent constitutive model, and hence, compute the corresponding material parameters of the model. The determined experimetal curves for IN718 at a strain rate of $0.1 \mathrm{~s}^{-1}$ for the different temperatures listed in Table 1 are presented in Fig.1B.

While the compression experiments were assumed to provide an homogeneous deformation field in the specimens (uniform strain and stress states), giving stress-strain data that are used for material model calibration, the upsetting tests of the double truncated cone specimens induced a strain gradient in the sample (non-uniform stress state), providing load-displacement curves that can be used for model validation. A sample of the loaddisplacement curves for IN718 obtained using Table 2 is presented in Fig.1C. Besides, these upsetting tests are commonly performed to obtain microstructural information for a range of strains within a single specimen. In particular, the experimental program planned to use different dwell times before quenching to affect the grain morphology developed in the deformed upset specimens, information that is mainly used to develop / validate microstructural models of static recrystallization and grain growth. This aspect is not addressed in this work, and we mainly use the load-displacement curves for model validation.

\section{An Internal State Variable Material Model - EMMI}

Modeling and simulation of thermo-mechanical processing require the use of constitutive models that, for complex deformation histories, can accurately predict the evolution of 
Table 1. Test Matrix for Uniaxial Compression Experiments

\begin{tabular}{|ccc|}
\hline Material & Temperature $\theta,{ }^{\circ} \mathrm{C}\left({ }^{\circ} \mathrm{F}\right)$ & Strain Rate $\dot{\epsilon}, \mathrm{s}^{-1}$ \\
\hline \hline IN718, IN718Plus & $982(1800)$ & $0.1,1.0$ \\
& $1037(1900)$ & $0.1,1.0$ \\
& $1093(2000)$ & $0.1,1.0$ \\
\hline
\end{tabular}

Table 2. Test Matrix for Double Cone Compression Experiments, $\dot{\epsilon}=0.5 \mathrm{~s}^{-1}$

\begin{tabular}{|cr|}
\hline Material & Temperature, ${ }^{\circ} \mathrm{C}\left({ }^{\circ} \mathrm{F}\right)$ \\
\hline \hline IN718 & $996(1825)$ \\
& $1037(1900)$ \\
\hline IN718Plus & $982(1800)$ \\
& $1024(1875)$ \\
& $1065(1950)$ \\
\hline
\end{tabular}

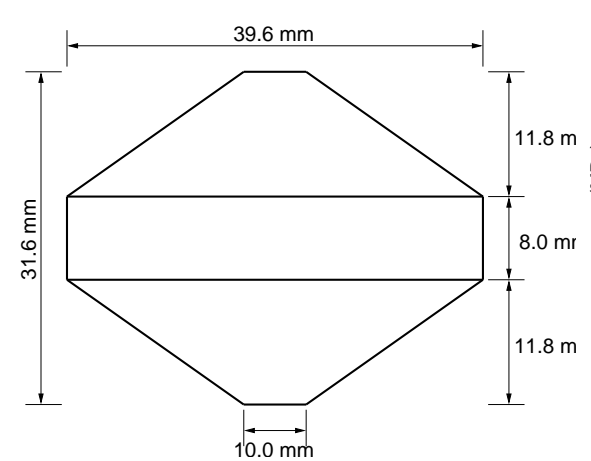

A

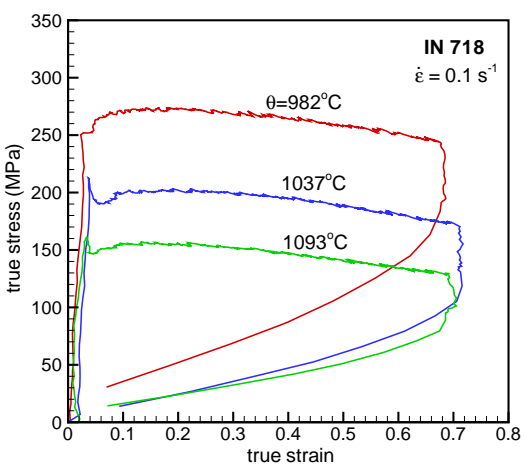

$B$

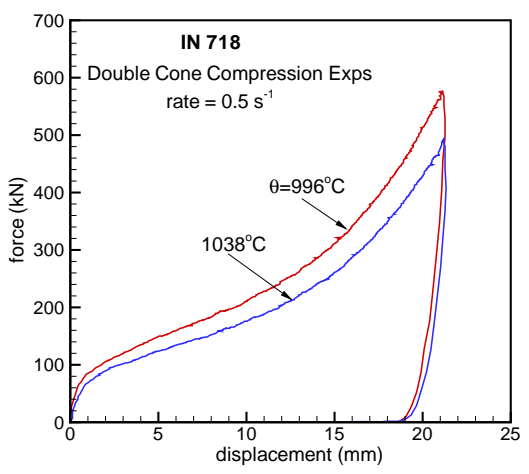

$C$

Figure 1. Double cone specimen geometry and mechancial response from uniaxial and double cone compression tests for IN718.

main microstructural features typically observed during the high temperature deformation of metals. Among these features are dislocation hardening, static/dynamic recovery processes, static/dynamic recrystallization, and grain growth, among others. In this context, the Evolving Microstructural Model of Inelasticiy or EMMI [24], is a physically-based internal state variable model that can be used to describe the rate- and temperature-dependent finite deformation behavior of metals. Although the current version of the model mainly accounts for thermally activated dislocation motion (kinetic equation) and hardening laws with dislocation storage/recovery processes, current efforts are underway to extend the model and include recrystallization and grain growth effects.

A main feaure of EMMI is that the constitutive equations are written in a fully dimensionless form by choice of appropriate scaling parameters. All stress-like quantities are scaled by twice the shear modulus, except for pressure which scales with bulk modulus. The characteristic length for normalization is the magnitude of the Burger's vector of the underlying crystals. Temperatures are scaled by the melting temperture. A characteristic time is introduced by considering the diffusivity of defects through the crystal at the melt temperature. The normalization allows the use of a small number of parameters that define the scale for the problem (moduli, mass density, Burger's vector, melt temperture, etc), with the remainder of the parameters being dimensionless. It is important to note that the use of normalized equations may play an important role in extrapolating the fitted response of a 
particular material to other similar materials. In addition, using a dimensionless model reduces the constitutive equations to a simpler form, simplifies the fitting parameter procedure and increases the robustness of the numerical implementation of the model.

The basic formulation of the plasticity and temperature aspects of the model relies on an extended description of the large deformation kinematics using the multiplicative decompositon of the deformation gradient into thermal, plastic and elastic components. This kinematics is then coupled with a thermodynamic approach with internal state variables, as proposed in [25]. A detailed formulation of the model including isotropic damage can be found elsewhere [24]. Here, we mainly list the plasticity version of the constitutive equations of EMMI written in dimensionless form in the so-called current configuration. Some particular symbols used to write the EMMI equations are: $\breve{a}$ to denote a dimensionless quantity, $\stackrel{*}{a}$ to represent a dimensionless time derivative, and $\breve{a}$ (bold-faced letter) to denote a dimensionless second-rank tensor. The specific version of the equations presented below is valid for small elastic strains (typical in metals), isotropic plasticity, and isotropic thermal expansion:

$$
\begin{aligned}
& \text { kinematics: }
\end{aligned}
$$

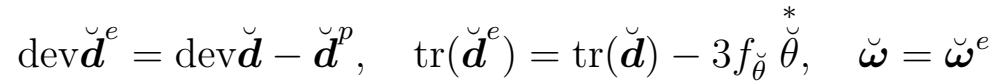

$$
\begin{aligned}
& \text { elastic law: } \\
& \breve{\mathcal{L}}_{v}^{e} \operatorname{dev} \breve{\boldsymbol{\tau}}=\operatorname{dev} \breve{\boldsymbol{d}} \\
& \breve{p}_{\tau}^{*}=\operatorname{tr}\left(\breve{\boldsymbol{d}}^{e}\right) \\
& \text { flow rule: } \\
& \breve{\boldsymbol{d}}^{p}=\sqrt{\frac{3}{2}} \stackrel{\stackrel{*}{\epsilon}}{\stackrel{\sim}{\epsilon}} \breve{\boldsymbol{n}}, \quad \stackrel{\sim}{\epsilon}^{p}=\breve{f}\left[\sinh \left(\left\langle\frac{\breve{\sigma}_{e q}}{\breve{\kappa}_{s}+\breve{Y}}-1\right\rangle\right)\right]^{\breve{n}} \\
& \text { hardening rules: } \\
& \breve{\mathcal{L}}_{v}^{e} \breve{\boldsymbol{\alpha}}=\breve{h} \breve{\boldsymbol{d}}^{p}-\breve{r}_{d} \stackrel{\breve{\epsilon}}{ }^{p} \sqrt{\frac{2}{3}}\|\breve{\boldsymbol{\alpha}}\| \breve{\boldsymbol{\alpha}} \\
& \stackrel{*}{\kappa}_{s}=\left(\breve{H}-\breve{R}_{D} \breve{\kappa}_{s}\right) \stackrel{\stackrel{*}{\epsilon}}{\mathrm{\epsilon}}-\breve{R}_{S} \breve{\kappa}_{s} \sinh \left(\breve{Q}_{S} \breve{\kappa}_{s}\right)
\end{aligned}
$$

with

$$
\breve{\boldsymbol{n}}=\sqrt{\frac{3}{2}} \frac{\breve{\boldsymbol{\xi}}}{\breve{\sigma}_{e q}}, \quad \breve{\sigma}_{e q}=\sqrt{\frac{3}{2}}\|\breve{\boldsymbol{\xi}}\|, \quad \breve{\boldsymbol{\xi}}=\operatorname{dev} \breve{\boldsymbol{\tau}}-\frac{2}{3} \breve{\boldsymbol{\alpha}}
$$

where $\breve{\mathcal{L}}_{v}^{e}(\bullet)$ denotes a corrotational rate typically used to make the formulation frame indifference. In these equations, $\left(\breve{\boldsymbol{\tau}}, \breve{p}_{\tau}\right)$ are the total stress tensor and its hydrostatic part (pressure), $\left(\breve{\boldsymbol{d}}, \breve{\boldsymbol{d}}, \breve{d}^{p}\right)$ are the total, elastic and plastic rate of deformation tensors, $\left(\breve{\boldsymbol{w}}, \breve{\boldsymbol{w}}^{e}\right)$ are the total and elastic spin tensors, $\left(\breve{\boldsymbol{\alpha}}, \breve{\kappa}_{s}\right)$ are the internal state variables representing strengths for kinematic (tensor) and isotropic (scalar) hardening, $\breve{\theta}$ is temperature, and $f_{\breve{\theta}}$ is a function describing the thermal expansion characteristics of the material. The symbols

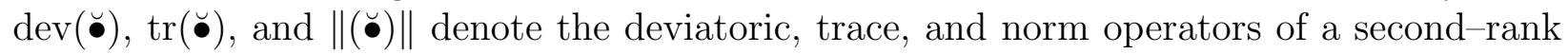
tensor.

The normalized temperature-dependent plasticity parameters of the model are $(\breve{f}, \breve{n}$, $\breve{Y}, \breve{r}_{d}, \breve{h}, \breve{R}_{D}, \breve{H}, \breve{R}_{S}, \breve{Q}_{S}$ ), and they are expressed in terms of other constants as given in Table 3. Commonly, these parameters are used to fit the predicted plastic behavior of the model to experimental stress-strain responses for a particular material obtained at different temperatures and strain rates. In particular, the function $\breve{Y}$, which represents the yield strength of the material, is expressed in terms of the nondimensional temperature-dependent function

$$
\widehat{\breve{Y}}(\breve{\theta})=\frac{\breve{m}_{1}}{1+\breve{m}_{2} \exp \left(\frac{-\breve{m}_{3}}{\breve{\theta}}\right)} \frac{1}{2}\left[1+\tanh \left(\breve{m}_{4}\left(\breve{m}_{5}-\breve{\theta}\right)\right)\right]
$$


where $\breve{m}_{1}, \breve{m}_{2}, \breve{m}_{3}, \breve{m}_{4}$, and $\breve{m}_{5}$ are additional material constants. Also, the shear modulus, $\mu(\breve{\theta})$, and bulk modulus, $\mathcal{K}(\breve{\theta})$, follow a linear dependence on temperature as indicated by the non-dimensional functions

$$
\widehat{\mu}(\breve{\theta})=\mu(\breve{\theta}) / \mu_{0}=1+c_{\theta \mu}\left(\breve{\theta}-\breve{\theta}_{0}\right), \quad \widehat{\mathcal{K}}(\breve{\theta})=\mathcal{K}(\breve{\theta}) / \mathcal{K}_{0}=1+c_{\theta \mathcal{K}}\left(\breve{\theta}-\breve{\theta}_{0}\right)
$$

where $\left(c_{\theta \mu}, c_{\theta \mathcal{K}}\right)$ are material constants, $\breve{\theta}_{0}$ is a reference temperature, and $\left(\mu_{0}, \mathcal{K}_{0}\right)$ are the shear and bulk moduli at $\breve{\theta}_{0}$.

Table 3. Nondimensional Material Parameters of EMMI Model.

\begin{tabular}{|c|c|c|}
\hline$\stackrel{*}{\stackrel{*}{\epsilon}^{p}}$-equation & $\stackrel{*}{\breve{\alpha}}$-equation & $\stackrel{*}{\kappa}_{s}$-equation \\
\hline 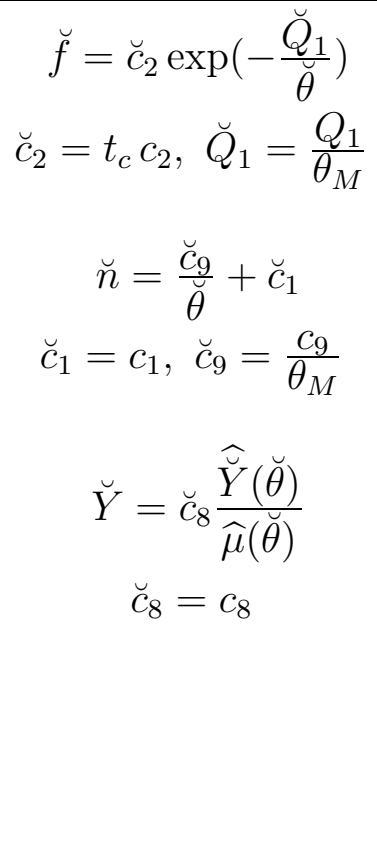 & $\begin{array}{c}\breve{r}_{d}=\breve{c}_{3} \exp \left(-\frac{\breve{Q}_{2}}{\breve{\theta}}\right) \\
\breve{c}_{3}=c_{3}, \breve{Q}_{2}=\frac{Q_{2}}{\theta_{M}} \\
\breve{h}=\breve{c}_{4} \\
\breve{c}_{4}=c_{4}\end{array}$ & $\begin{array}{c}\breve{R}_{D}=\breve{c}_{5} \exp \left(-\frac{\breve{Q}_{3}}{\breve{\theta}}\right) \\
\breve{c}_{5}=c_{5}, \breve{Q}_{3}=\frac{Q_{3}}{\theta_{M}} \\
\breve{H}=\breve{c}_{6} \\
\breve{c}_{6}=c_{6} \\
\breve{R}_{S}=\breve{c}_{7} \exp \left(-\frac{\breve{Q}_{4}}{\breve{\theta}}\right) \\
\breve{c}_{7}=t_{c} c_{7}, \quad \breve{Q}_{4}=\frac{Q_{4}}{\theta_{M}} \\
\breve{Q}_{S}=\breve{c}_{10} \exp \left(-\frac{\breve{Q}_{5}}{\breve{\theta}}\right) \\
\breve{c}_{10}=c_{10}, \breve{Q}_{5}=\frac{Q_{5}}{\theta_{M}}\end{array}$ \\
\hline
\end{tabular}

In the above model equations, there are 20 material constants that need be determined from experimental data. These are:

$$
\breve{a}_{k}=\left[\breve{c}_{1}, \breve{c}_{2}, \breve{c}_{3}, \breve{c}_{4}, \breve{c}_{5}, \breve{c}_{6}, \breve{c}_{7}, \breve{c}_{8}, \breve{c}_{9}, \breve{c}_{10}, \breve{Q}_{1}, \breve{Q}_{2}, \breve{Q}_{3}, \breve{Q}_{4}, \breve{Q}_{5}, \breve{m}_{1}, \breve{m}_{2}, \breve{m}_{3}, \breve{m}_{4}, \breve{m}_{5}\right]
$$

The current version of the model, assumes $\breve{Q}_{S}=1\left(\breve{c}_{10}=1, \breve{Q}_{5}=0\right)$, and hence the set $\breve{a}_{k}$ reduces to 18 material parameters. To compute these parameters, we will first determine $\breve{m}_{1}$ to $\breve{m}_{5}$ by fitting Eq. (2) to reported yield strength-temperature data. These values will then be used to determine the other 13 parameters by fitting the predicted stress response of the model to experimental compression stress-strain data. This fitting is performed using EMMI-Fit, a matlab code that implements the reduced one-dimensional equations of the model together with a constrained minimization problem based on a discrete nonlinear least square functional that tries to minimize the distance between the model predictions and the experimental data.

To illustrate the predictive capability of EMMI, we present in Fig.2 the summary of the model calibration to both reported yield strength-temperature data [26] and experimental strain-stress curves for stainless steel 304L [21].

\section{Modeling the Mechanical Response of IN718 and IN718Plus}

In this section, the EMMI constitutive equations are applied to model the mechanical response of the nickel-based superalloys IN718 and IN718Plus. The model material parameters 

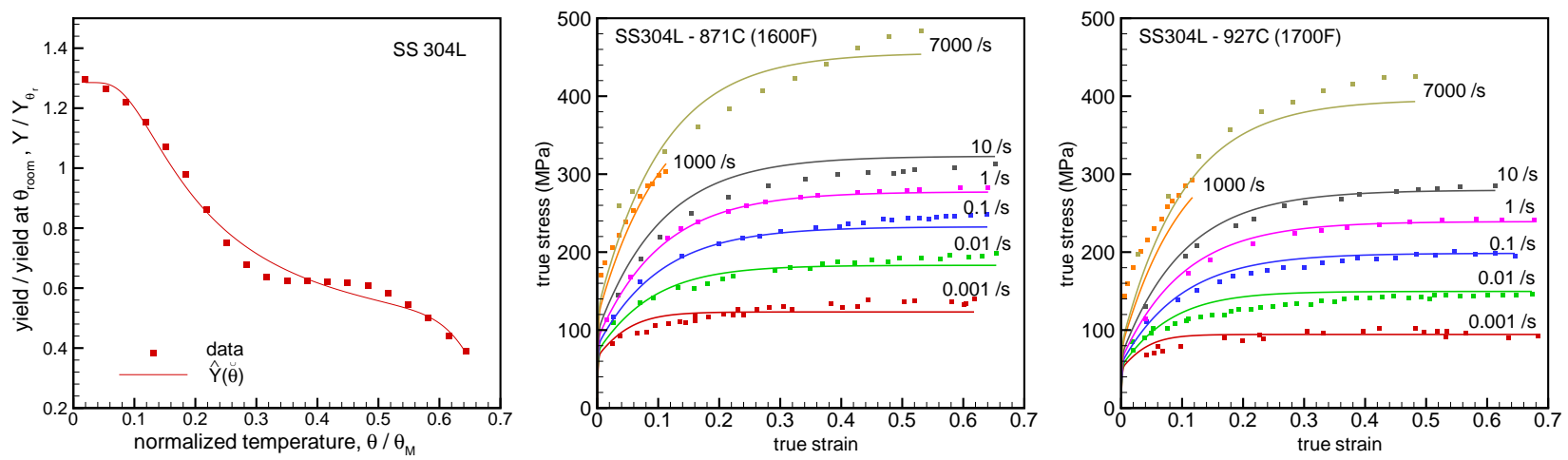

Figure 2. Yield function and EMMI model response fitted to experimental data for SS304L. are determined by correlating yield strength-temperature curves obtained from published data, and the strain-stress responses generated from the isothermal compression tests on cylindrical specimens. The validation of the model is performed using finite element simulations to predict the experimental load-displacement curves from the truncated doule cone compression experiments. The value of the physical/mechanical properties used to normalize (scale) the EMMI equations for these superalloys is given in Table 4. These properties have been obtained from a number of sources $[27,28]$, and have been assumed to be the same for both superalloys.

The first step of the calibration procedure fits Eq. (2) to the yield strength-temperature data shown in Fig.3. This fit for both IN718 and IN718Plus is presented in the same figure, while the corresponding computed $\breve{m}_{i}$ parameters are given in Table 5 . Next, using the computed $\breve{m}_{i}$ parameters, the EMMI model is calibrated to strain-stress data determined from the isothermal compression tests at different temperatures/strain rates. EMMI-Fit is used for this purpose. The fitted response to this experimental data is presented in Fig. 4 for IN718 and Fig.5 for IN718Plus, and the respective computed material constants of EMMI are given in Table 6. As can be noted from these figures, the experimental strain-stress curves show a softening response that the current version of the model does not capture. In general, this softening may be due to adiabatic heating and/or dynamic recrystallization phenomena. Current efforts are underway to consider both effects in the constitutive framework.

Table 4. Physical/Mechanical Properties for IN718 / IN718Plus.

\begin{tabular}{|cccc|}
\hline Property & Notation & Value & Units \\
\hline \hline Burgers Vector & $b$ & $2.5 \times 10^{-10}$ & $\mathrm{~m}$ \\
Melting Temperature & $\theta_{M}$ & 1573 & $\mathrm{~K}$ \\
Shear Modulus $(\mu)$ at $300 K$ & $\mu_{0}$ & $7.86 \times 10^{4}$ & $\mathrm{MPa}$ \\
Temperature dependence of $\mu$ & $c_{\theta \mu}$ & -0.50 & - \\
Bulk Modulus $(\mathcal{K})$ at $300 K$ & $\mathcal{K}_{0}$ & $16.09 \times 10^{4}$ & $\mathrm{MPa}$ \\
Temperature dependence of $\mathcal{K}$ & $c_{\theta \mathcal{K}}$ & -0.36 & - \\
Lattice Diffusion (prefactor) & $D_{0 \nu}$ & $1.6 \times 10^{-4}$ & $\mathrm{~m}^{2} / \mathrm{s}$ \\
Lattice Diffusion (activation energy) & $Q_{\nu}$ & $285 \times 10^{3}$ & $\mathrm{~J} / \mathrm{mole}$ \\
\hline
\end{tabular}

The numerical simulation of the isothermal hot upsetting tests for the double-cone shape specimens has been performed using ABAQUS/explicit and a VUMAT that implements the numerical integration of the EMMI constitutive equations. The detailed geometry of the specimen is presented in Fig.1A. Due to symmetry in both loading and geometry the analysis is performed as a two-dimensional axi-symmetric problem, with only one-half of the 
Table 5. Parameters $\breve{m}_{i}$ of Yield Function $\widehat{\breve{Y}}(\breve{\theta})$.

\begin{tabular}{|cccccc|}
\hline Material & $\breve{m}_{1}$ & $\breve{m}_{2}$ & $\breve{m}_{3}$ & $\breve{m}_{4}$ & $\breve{m}_{5}$ \\
\hline \hline IN718 & 1.2321 & 0.45080 & 0.14395 & 11.490 & 0.67071 \\
IN718Plus & 1.2240 & 0.44455 & 0.15452 & 15.625 & 0.69911 \\
\hline
\end{tabular}

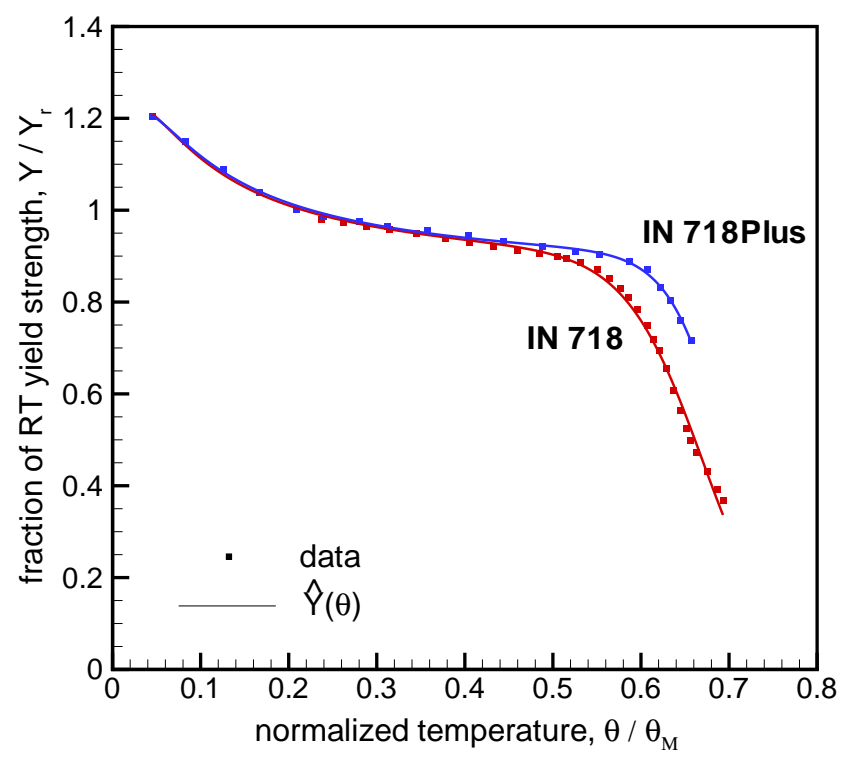

Figure 3. Fit to temperature dependent yield strength for both IN718 and IN718Plus.

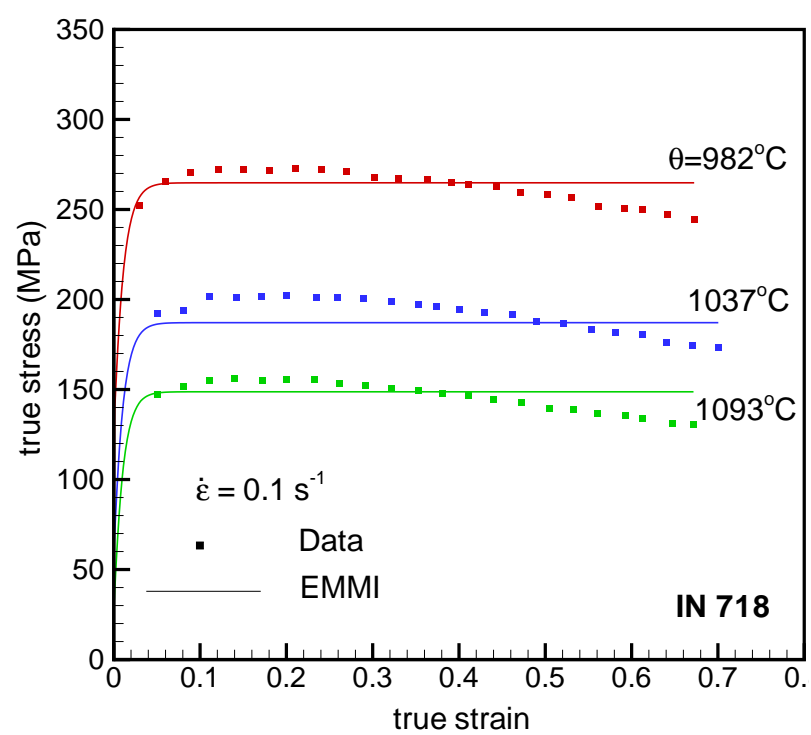

$(A)$

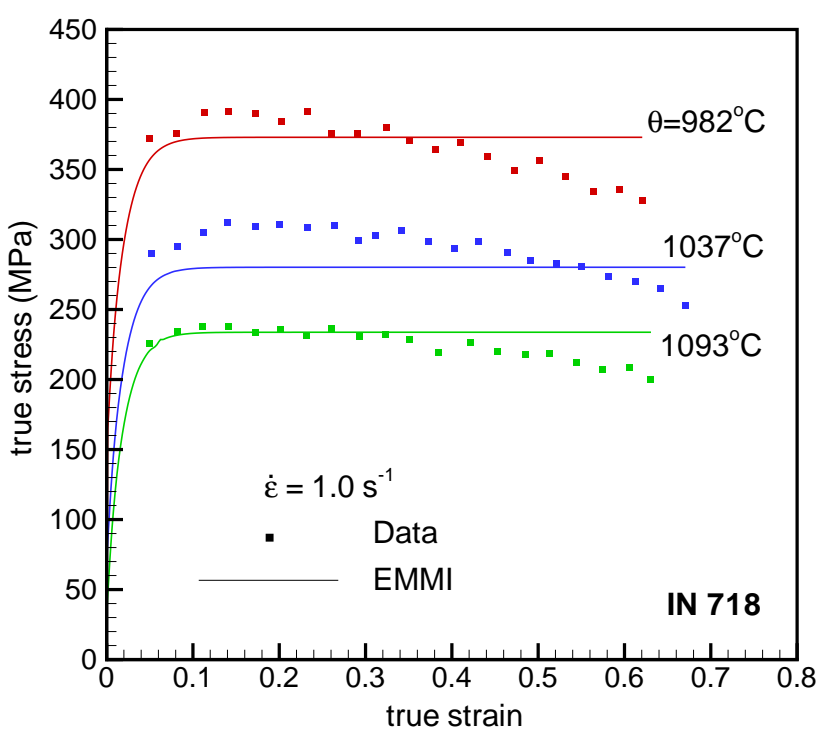

$(B)$

Figure 4. EMMI fitted strain-stress response of nickel-based superalloy IN718 for strain rates of (A) $\dot{\epsilon}=0.1 \mathrm{~s}^{-1}$ and (B) $\dot{\epsilon}=1.0 \mathrm{~s}^{-1}$.

specimen being considered. The specimen is discretized with 539 axi-symmetic elements, ABAQUS type CAX4R (reduced-integration element with one integration point), see Fig.6A. Symmetry boundary conditions are imposed along $\mathrm{x}=0$ and $\mathrm{y}=0$, while a displacement boundary condition is applied incrementally at the top surface along the -y direction through 


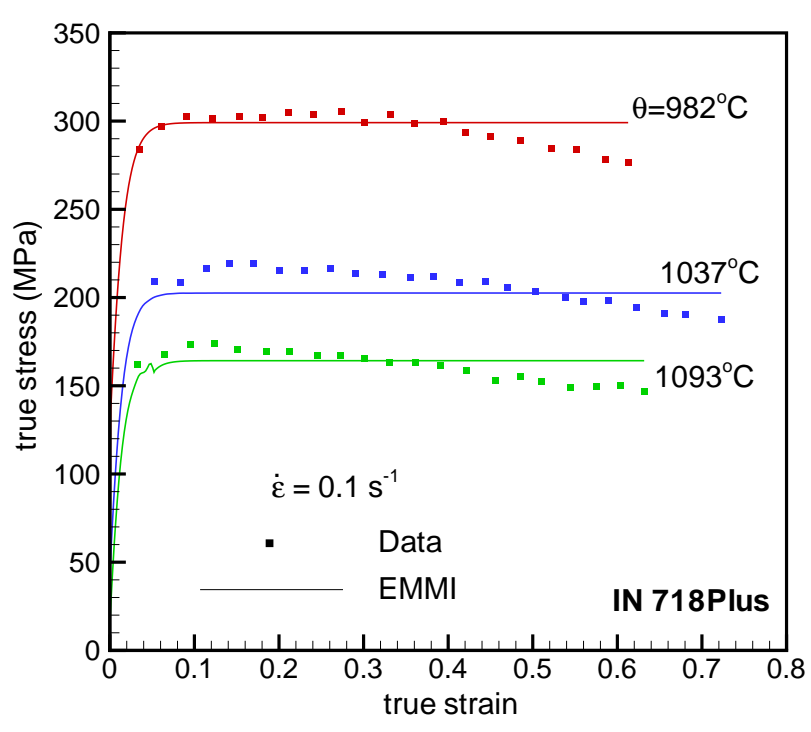

(A)

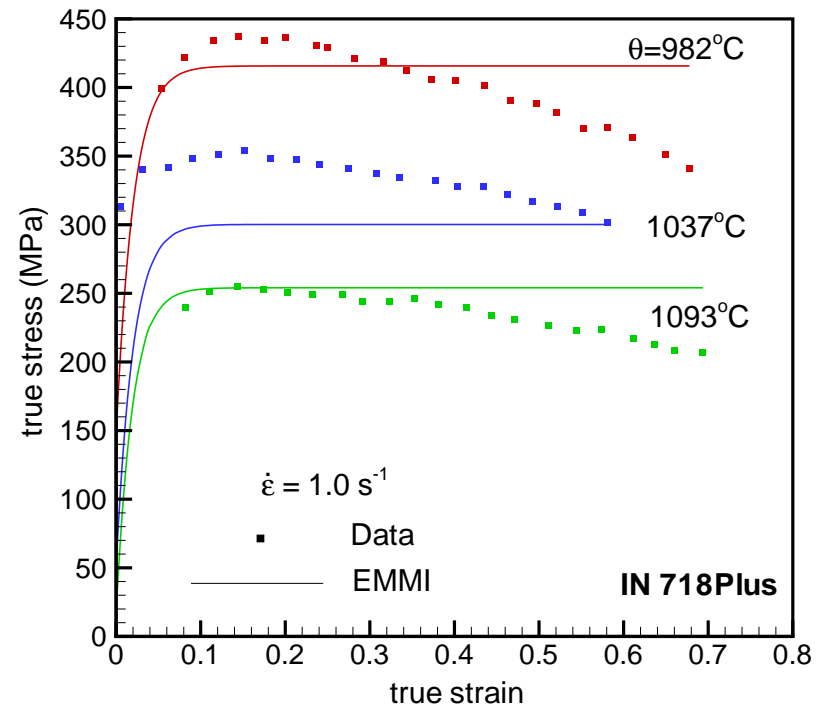

(B)

Figure 5. EMMI fitted strain-stress response of nickel-based superalloy IN718Plus for strain rates of (A) $\dot{\epsilon}=0.1 \mathrm{~s}^{-1}$ and (B) $\dot{\epsilon}=1.0 \mathrm{~s}^{-1}$.

Table 6. Non-Dimensional EMMI Material Constants.

\begin{tabular}{|ccc|}
\hline Constant & IN718 & IN718Plus \\
\hline \hline$\breve{c}_{1}$ & 0.93490 & 0.98396 \\
$\breve{c}_{2}$ & $6.6213 \times 10^{-11}$ & $7.9600 \times 10^{-11}$ \\
$\breve{c}_{3}$ & $7.5057 \times 10^{5}$ & $1.5765 \times 10^{6}$ \\
$\breve{c}_{4}$ & $3.2447 \times 10^{-2}$ & $5.8655 \times 10^{-3}$ \\
$\breve{c}_{5}$ & 66.367 & 88.961 \\
$\breve{c}_{6}$ & $1.5476 \times 10^{-2}$ & $1.9041 \times 10^{-2}$ \\
$\breve{c}_{7}$ & $5.5359 \times 10^{-2}$ & $1.0838 \times 10^{-1}$ \\
$\breve{c}_{8}$ & $4.2081 \times 10^{-3}$ & $4.5186 \times 10^{-3}$ \\
$\breve{c}_{9}$ & 1.3952 & 1.0964 \\
$\breve{Q}_{1}$ & 0.24402 & 0.25901 \\
$\breve{Q}_{2}$ & $1.3355 \times 10^{-2}$ & $4.6295 \times 10^{-2}$ \\
$\breve{Q}_{3}$ & 0.31148 & 0.50214 \\
$\breve{Q}_{4}$ & 0.97489 & 2.1887 \\
\hline
\end{tabular}

a rigid die such that a constant deformation rate of $\dot{\epsilon}=0.5 \mathrm{~s}^{-1}$ is obtained. The total simulation time is $T=2.2 s$. The displacement history is computed using $u=h_{0} / 2[\exp (\dot{\epsilon} t)-1]$, where $h_{0}$ is the initial height of the specimen, $\dot{\epsilon}$ is the applied strain rate, and $\mathrm{t}$ is time $(0 s \leq t \leq 2.2 s)$.

The simulations are performed under isothermal conditions, at two temperatures for IN718 and three temperatures for IN718Plus, as indicated by Table 2. The stress-strain response at each integration point is given by the EMMI predictions using the material properties listed in Table 6. A "rough" friction condition (ABAQUS terminology for a rigid sticking condition) is assumed between the rigid die and specimen. A typical time step in the simulations is on the order of $10^{-8} \mathrm{~s}$, giving much more than 1,000,000 time increments needed to solve each case. Mass scaling with a factor of 10 is used to speed up the simulation runs. 
A typical deformed finite element mesh is presented in Fig.6B. This case is for the IN718Plus double cone specimen deformed at $982^{\circ} \mathrm{C}$. The contour plots shown indicates levels of equivalent plastic strain. Clearly, the deformation field is rather non-homogeneous, indicating strain gradients across the specimen, in particular for points in the middle of the specimen (e.g. point 1), between the free surface and the center of the specimen.

A comparison of the computed force-displacement curves with the experimental ones for both superalloys is presented in Fig.7. The predicted response agrees well with the experiments at low deformation levels, however, the computed values start to deviate from the data as the deformation increases, resulting in an overpredicted load level at large deformations. It is believed that this difference may be attributed to the missing features in the material model to predict the softening effect of annealing phenomena (recrystallization and grain growth) on the macrocopic stress response of the material points (integration points) in the finite element simulations.

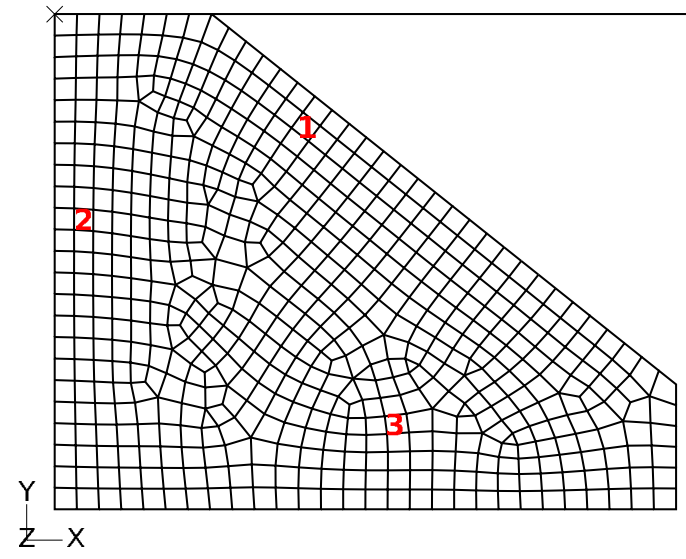

$(A)$

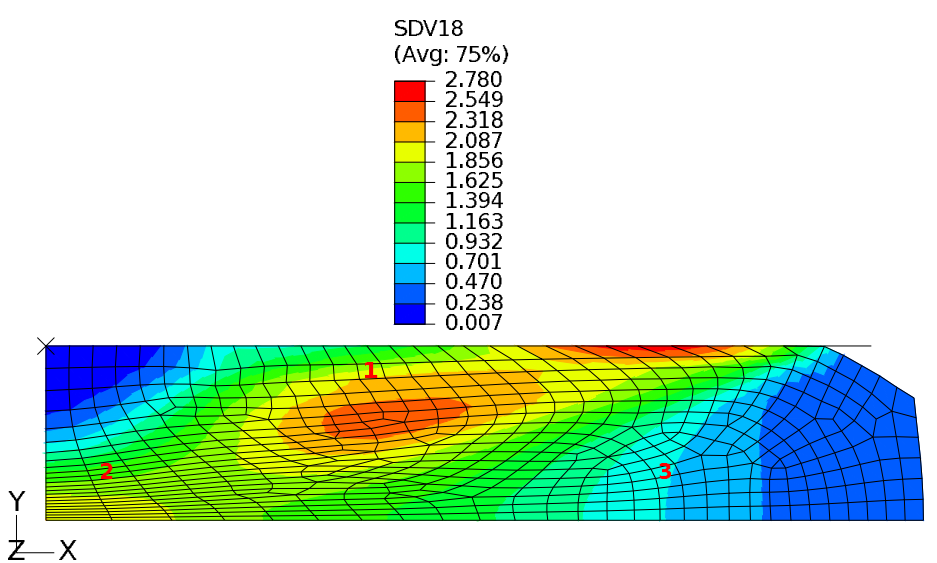

$(B)$

Figure 6. (A) Finite element mesh for axisymmetric double cone specimen. (B) Deformed double cone specimen showing contours of equivalent plastic strain (ABAQUS variable SDV18) for IN718Plus at $982^{\circ} \mathrm{C}$.

\section{Summary}

This work has presented the application of the internal state variable material model EMMI to describe the rate- and temperature-dependent response of IN718 and IN718Plus, two Nickel-based superalloys used to manufacture components of aero-engines. The model, calibrated using strain-stress data from isothermal compression tests, has been validated using load-displacement curves from double cone isothermal upsetting tests at various temperatures. In general, the model captures the stress/load levels of the tests at lower deformation levels as well as the rate and temperature dependence of the mechanical response of these superalloys. However, specific details of this behaviour, such as the stress softening due recrystallization and grain growth are not yet represented in the model. Ongoing work is focussed on enhancing EMMI with microstructural models to capture the effect of these annealing phenomena on the macroscopic strain-stress response. 


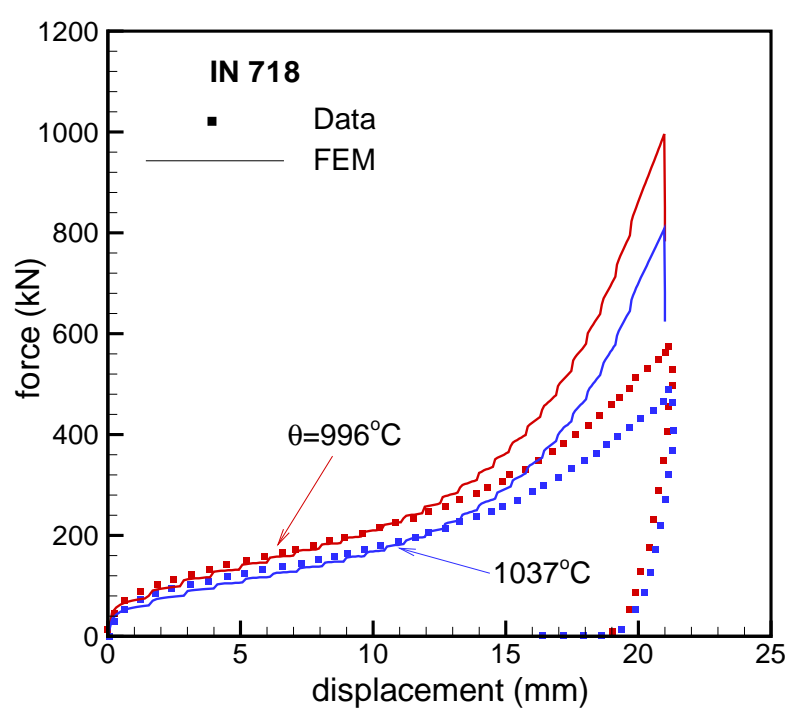

$(A)$



$(B)$

Figure 7. The isothermal load-displacement curves for the double cone compression tests for both (A) IN718 and (B) In718Plus: comparison of experimental data and numerical predictions.

\section{Acknowledgments}

This work was supported by the Center of Advanced Vehicular Systems (CAVS), Mississippi State University (MSU), and the Miltec Research and Technology (MRT), Inc.

\section{References}

1. Bergstrom, D. S. and Bayha, T. D., (2005), "Properties and Microstructure of Allvac 718Plus Alloy Rolled Sheet," Sixth International Special Emphasis Symposium on Superalloys 718, 625, 707 and Derivatives, ed. E. A. Loria, Pittsburgh, PA, October, 243-252.

2. Cao, W. D., and Kennedy, R. L., (2005) "New Developments in Wrought 718-Type Superalloys", Acta Metall. Sinica, 18:39-46.

3. Xie, X., Xu, C., Wang, G., Dong, J., Cao, W. D., Kennedy, R., (2005) "TTT Diagram of a Newly Developed Nickel-Base Superalloys-Allvac 718Plus", Superalloys 718, 625, 706 and Derivatives, Ed. E. A. Loria, TMS.

4. Du, J. H., Lu, X. D., Deng, Q., Qu, J. L., Zhuang, J. Y., and Zhong, Z. Y, (2007), "HighTemperature Structural Stability and Mechanical Properties of Novel 718 Superalloy", Mat. Sci. Eng.: A, 452-453:584-591.

5. Maziasz, P. J., Wright, I. G., Shingledecker, T. B., Gibbons, T. B., Romanowsky, R. R., (2005) "Defining of the Materials Issues and Research for Ultrasupercritical Steam Turbines", Proceedings to the Fourth International Conference on Advances in Materials Technology For Fossil Power Plants, Hilton Head, SC, Oct. 25-28.

6. Kemanpur, A., Lee, P. D., Tin, S, and McLean, M. (2005) "Integrated Model for Tracking Defects through Full Manufacturing Route of Aerospace Discs", Mater. Sci. Tech., 21:437444. 
7. Callister, Jr, W. D., (2000) "Materials Science and Engineering, An Introduction", John Wiley \& Sons, Inc.

8. Humphreys, F. J. and Hatherly, M. (1996) "Recrystallization and Related Annealing Phenomena", Pergamon, Elsevier Science Ltd.

9. Zhou, L. X. and Baker, T. N., (1995), "Effects of Dynamic and Metadynamic Recrystallization on Microstructure of Wrought IN718 due to Hot Deformation", Mat. Sci. Eng., A196:89-95.

10. Brand, A. J., Karhausen, K., and Kopp, R, (1996), "Microstructural Simulation of Nickel-Based Alloy Inconel 718 in Production of Turbine Discs", Mat. Sci. Tech., 12:963969.

11. Medeiros, S. C., Prasad, Y. V. R. K., Frazier, W. G., Srinivasan, R., (2000) "Microstructural Modeling of Metadynamic Recrystallization in Hot Working of IN718 Superalloy", Mat. Sci. Eng., A293:198-207.

12. Jo, C.-Y.), Joo, D.W., and Kim, I.-B., (2001), "Precipitation adn Deformation Behavior of Cast Alloy 718 during Creep and Thermal Exposure", Mater. Sci. Tech., 17:1191-1196.

13. Semiatin, S. L., Weaver, D. S., Fagin, P. N., Glavicic, M. G., Goetz, R. L., Frey, N. D., Kramb, R. C., and Antony, M. M., (2004), "Deformation and Recrystallization Behavior during Hot Hot Working of a Coarse-Grain, Nickel-Base Superalloy Ingot Material", Metall. Mat. Trans., 35A:679-693.

14. Guest, R. P. and Tin, S., (2005), "Modelling Microstructural Transformations of Nickel Base Superalloy IN718 during Hot Deformation", 6th Int. Symposium on Superalloys 718, 625, 706 and their Derivatives, 385-397.

15. Weaver, D. S., and Semiatin, S. L., (2007), "Recrystallization and Grain-Growth Behavior of a Nickel-Base Superalloy during Multi-Hit Deformation", Scripta Materialia, 57:10441047.

16. Srinivasan, R., Ramnarayan, V., Deshpande, U., Jain, V, weiss, I., (1993), "Computer SImulation of the Forging of Fine Grain IN-718 Alloy", Metall. Trans. A, 24A:2061-2069.

17. Huang, D., Wu, W. T, Lambert, D., and Semiatin, S. L., (2001), "Computer Simulation of Microstructure Evolution during Hot Forging of Waspalloy and Nickel Alloy 718" in Microstructure Modeling and Prediction During Thermomechanical Processing, 137-146, Nov $4-8$, Indianapolis, ID.

18. Bammann, D. J., (1990), "Modeling Temperature and Strain Rate Dependent Large Deformations of Metals," Appl. Mech. Rev., 1:312-318.

19. Manonukul, A., and Dunne, F. P. E., (2006) "Effect of Inhomogenous Deformation on Initiation of Dynamic Recrystallization" Proceddings of the fourth International Conference on Recrystallization and Related Phenomena, Ed. T. Sakai and H.G. Suzuki, 13:223-228.

20. Lin, J., and Yang, J., (2007), "GA-Based Multiple Objective Optimization for Determining Viscoplastic Constitutive Equations for Superplastic Alloys", Int. J. Plast., 15:11811196.

21. Chiesa, M. L., Brown, A. A., Antoun, B. A., Ostien, J. T., Regueiro, R. A., Bammann, D. J., and Yang, N. Y. C., (2004) "Prediction of Final Material State in Multi-Stage Forging 
Processes", AIP Conference Proceedings, 712:510-515.

22. Lin, Y. P., Lin, J., Dean, T. A., and Brown, P. D., (2007), "Modelling of Viscoplastic Behavior of IN718 under Hot Forging Conditions", 10th ESAFORM Conference on Material Forming, AIP Conference Proceedings, 907:1199-1204

23. Costley, R. D., and Jones, M. K., (2008), "Optimal Design of Material Processes," Final Report, AFRL-RX-WP-TR-2008-4155.

24. Marin, E. B., Bammann, D. J., Regueiro, R. A., Johnson, G. C., (2006) "On the Formulation, Parameter Identification and Numerical Integration of the EMMI Model: Plasticity and Isotropic Damage" Sandia National Laboratories, CA, SAND2006-0200.

25. Coleman, B., and Gurtin, M., (1967), "Thermodynamics With Internal State Variables," J. Chem. Phys., 47:597-613.

26. Department of Defense Handbook, (2003), "Metallic Materials and Elements for Aerospace Vehicles Structures," MIL-HDBK-5J, January 31.

27. Frost, H., and Ashby, M., (1982), "Deformation Mechanism Maps," Pergamon Press.

28. Jeniski, Jr, R. A. and Kennedy, R. L. (2006) "Development of ATI Allvac 718Plus Alloy and Applications", Second Symposium On Recent Advances of Nb-Containing Materials in Europe. Also located at: http://www.allvac.com/allvac/718Plus. 